\title{
Aggressive Angiomyxoma Pelvis Presenting as an Ovarian Tumor with Vault Prolapse
}

\author{
Sujata Siwatch, Subhas Chandra Saha, Neelam Aggarwal, Lakhbir Dhaliwal, Raje Nijhawan
}

\section{ABSTRACT}

Background: Angiomyxoma is a rare tumor arising from the pelvis. Preoperative diagnosis and treatment may be difficult.

Case report: We report a rare case of aggressive angiomyxoma pelvis that presented as vault prolapse and was misdiagnosed as ovarian tumor.

Conclusion: As surgery is the main treatment, preoperative diagnosis and assessment of extent of the tumor for planning surgery cannot be overemphasized.

Synopsis: Angiomyxoma is a rare pelvic tumor with varied presentation. Preoperative diagnosis and assessment of extent of the tumor are important for its management.

Keywords: Angiomyxoma, Pelvic neoplasm, Soft tissue neoplasms.

How to cite this article: Siwatch S, Saha SC, Aggarwal N, Dhaliwal L, Nijhawan R. Aggressive Angiomyxoma Pelvis Presenting as an Ovarian Tumor with Vault Prolapse. J Postgrad Med Edu Res 2012;46(2):102-103.

\section{Source of support: Nil}

Conflict of interest: None declared

\section{CASE REPORT}

A 35-year-old lady presented with complaints of a mass coming out of introitus and abdominal distension for 10 years. She had a vaginal hysterectomy and some abdominal procedures done 10, 5 and 3 years ago. However, there were no records. There was no history of loss of appetite or weight lost. Examination showed a large nontender mass with restricted mobility filling the whole abdomen. Pelvic examination revealed vault prolapse and a right perineal bulge of $4 \times 4 \mathrm{~cm}$. Ultrasound showed a large solid soft tissue mass with few cystic areas and low echogenecity. Computerized tomography scan (CT) showed that the tumor extended from the pelvis to splenic flexure and along posterior abdominal wall, causing bilateral hydroureteronephrosis (Fig. 1). Fine needle aspiration cytology (FNA) of the mass showed low cellularity. Monomorphic ovoid cells suggestive of a spindle cell tumor were seen. CA125 was 417 IU.

An exploratory laparotomy was done along with the surgeon. A large lobulated, soft, gelatinous mass with solid and cystic areas filling the entire abdominal cavity was found. The left ovary was engulfed by the mass which extended into the left broad ligament, retroperitoneally into the pouch of douglas and into the right obturator fossa.
The mass was dissected retroperitoneally while tracing the ureters. Debulking was done, preserving the ureters, bladder and iliac vessels. Few presacral and right iliac vessels were injured. Extensive oozing was controlled by pressure, cautery and gel foam. The patient needed 10 units of blood transfusion. Due to excessive blood loss during surgery, diffuse infiltration of the tumor into the bladder and infralevator extension of the tumor, further debulking was defered and abdomen was closed after putting a drain. Postoperatively, she was found to have right ureteric injury at the level of pelvic brim, for which percutaneous nephrostomy was done. The histopathology revealed an edematous and loose myxoid stroma with few proliferative spindle shaped fibroblastic cells devoid of mitotic figures. These findings were suggestive of an aggressive angiomyxoma with involved margins (Fig. 2).

Postoperatively, CT scan done after 3 months showed residual pelvic tumor in the pelvis infiltrating the right ischiorectal fossa, bladder, anal canal, rectum and few small bowel loops. The prognosis and need for extensive abdominoperineal surgery for complete removal were discussed with the patient. She was planned for leuprolide injections that she could not afford. A repeat CT scan done at 5 months postoperatively showed no increase in size. The patient was on close follow-up with clinical examination and computerized tomography scan and was planned to undergo abdominoperineal resection in case of progression of disease. However, patient was lost to follow-up after 6 months of follow-up.

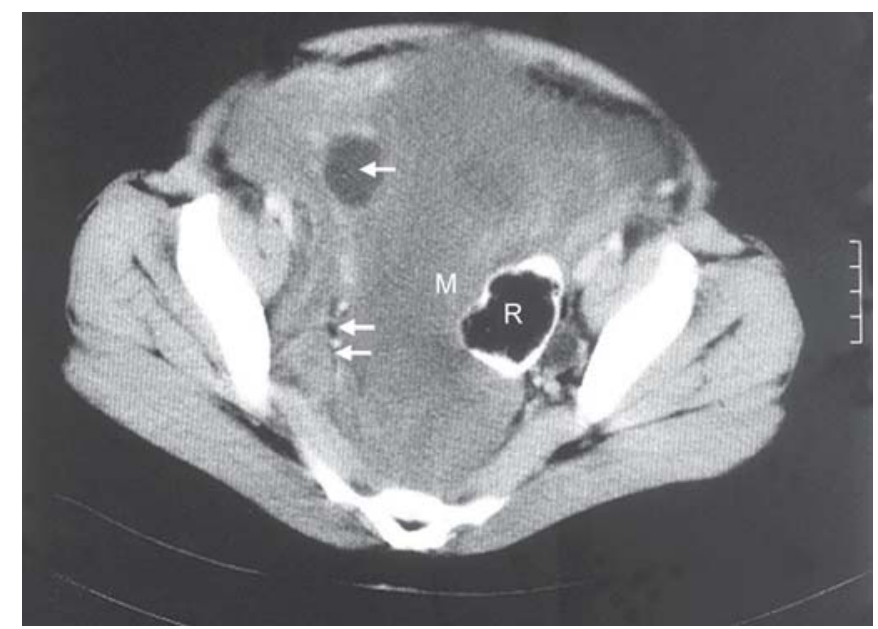

Fig. 1: CECT section through the pelvis shows heterogeneous mass (M) with cystic component (arrow). Rectum (R) is displaced to the left. Pelvic fat (double arrows) is seen engulfed in the mass 


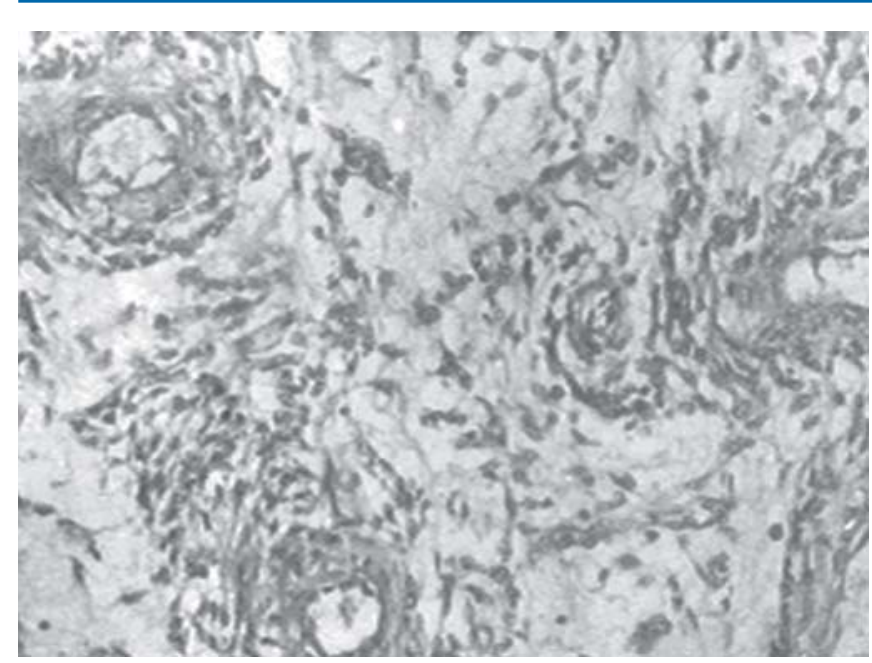

Fig. 2: Tissue section from the pelvis mass shows varying sized blood vessels surrounded by cells with ovoid to spindle nuclei and a myxoid stroma (hematoxylin and eosin $\times 280$ )

\section{DISCUSSION}

Angiomyxoma is a rare mesenchymal tumor arising from the soft tissues of pelvis or perineum that may present as a vulval mass, vaginal polyp, bartholin or a vaginal cyst. It occurs mainly in the reproductive age group. ${ }^{1}$ The size may vary from 1 to $60 \mathrm{~cm}$. The diagnosis can be made considering the clinical presentation aided by ultrasound, CT or MRI showing a hypodense mass with translevator extension, displacing rather than invading the pelvic organs. ${ }^{2}$ FNA reduces the diagnostic possibilities but histopathology alone gives the definite diagnosis. It has low cellularity with spindle shaped cells in a loose matrix containing medium sized arteries having fibrointimal proliferation. Most tumors have been found to have estrogen and progesterone receptor positive status. Tumor cells are at least focally immunoreactive to desmin, smooth muscle actin, muscle specific actin and vimetin. ${ }^{2}$

\section{CONCLUSION}

Surgery is the mainstay of treatment. ${ }^{1}$ The aim of surgery should be to excise as much as possible without causing urinary or anorectal dysfunction. Recurrence is local and reported in 36 to $72 \% .^{2}$ Radiotherapy and chemotherapy may not have much role due to the low mitotic activity seen. ${ }^{1}$ GnRH agonist ${ }^{3}$ and tamoxifen have been used successfully in few patients. The response can be assessed by clinical assessment, patient's symptomatology and radiographic findings.

\section{REFERENCES}

1. Chan YM, Hon E, Ngai SW, Ng TY, Wong LC. Aggressive angiomyxoma in females: Is radical resection the only option? Acta Obstet Gynecol Scand 2000 Mar;79(3):216-20.

2. Fetsch JF, Laskin WB, Lefkowitz M, Kindblom LG, MeisKindblom JM. Aggressive angiomyxoma: A clinicopathologic study of 29 female patients. Cancer 1996;78(1):79-90.

3. Behranwala KA, Thomas JM. Aggressive angiomyxoma: A distinct clinical entity. Eur J Surg Oncol 2003 Sep;29(7): 559-63.

\section{ABOUT THE AUTHORS}

\section{Sujata Siwatch}

Ex-Senior Resident, Department of Obstetrics, Gynecology and Pathology, Postgraduate Institute of Medical Education and Research Chandigarh, India

\section{Subhas Chandra Saha (Corresponding Author)}

Additional Professor, Department of Obstetrics, Gynecology and Pathology, Postgraduate Institute of Medical Education and Research Chandigarh-160012, India, e-mail: drscsaha@sify.com

\section{Neelam Aggarwal}

Assistant Professor, Department of Obstetrics, Gynecology and Pathology, Postgraduate Institute of Medical Education and Research Chandigarh, India

\section{Lakhbir Dhaliwal}

Professor, Department of Obstetrics, Gynecology and Pathology Postgraduate Institute of Medical Education and Research Chandigarh, India

\section{Raje Nijhawan}

Additional Professor, Department of Obstetrics, Gynecology and Pathology, Postgraduate Institute of Medical Education and Research Chandigarh, India 